\title{
Terapia Ocupacional en la vida real de los pacientes con lesiones neurológicas
}

María Cristina Tafur"

La rehabilitación del paciente neurológico en Estados Unidos, esta conceptualizada con base en el marco de referencia de la Orgañización Mundial de La Salud de deficiencia, incapacidad y minusvalía. (U.S. Department of Health and Human Services, 1995). (Ver figura 1).

Con base en esta conceptualización el paciente que sostiene un lesión neurológica puede seguir varios caminos en su proceso de rehabilitación. Por ejemplo, esta el paciente que rápidamente se devuelve a su casa pues no hay consecuencias de la lesión, o el polo opuesto, que es el paciente que termina en un hogar para enfermos teniendo atención de enfermería constante, pues el individuo requiere atención permanente.

Estos diversos caminos que puede seguir la rehabilitación del paciente neurológico, no están únicamente sujetos a la condición médica, sino especialmente al complejo aspecto psicosocial. Es aquí donde principalmente se siente el impacto de la seguridad social. Dependiendo del seguro médico que tenga el paciente, pueden tener acceso a cierto número de horas (generalmente 4 horas cuando es Medicare) para tener un ayudante en la casa que facilite las tareas de aseo personal, cocina, compras y visitas médicas, etc. Si es un seguro médico privado, generalmente no ofrece ayuda para la casa y tampoco hay tratamiento en centros de rehabilitación sub-agudos. Casi siempre los pacientes tienen acceso a rehabilitación en la casa, la

* Trabajo presentado en el XII Congreso Colombiano de Terapia Ocupacional. Santa Marta. Marzo/01. - Terapeuta Ocupacional. Maestría en Terapia Ocupacional, Universidad de Nueva York, estudiante de Ph.D en T.O., Universidad de Nueva York. Egresada Escuela Colombiana de Rehabilitación. Universidad del Rosario. 
Fịgura 1: Diagrama de flujo de la rehabilitación del paciente con enfermedad cerebro váscular.

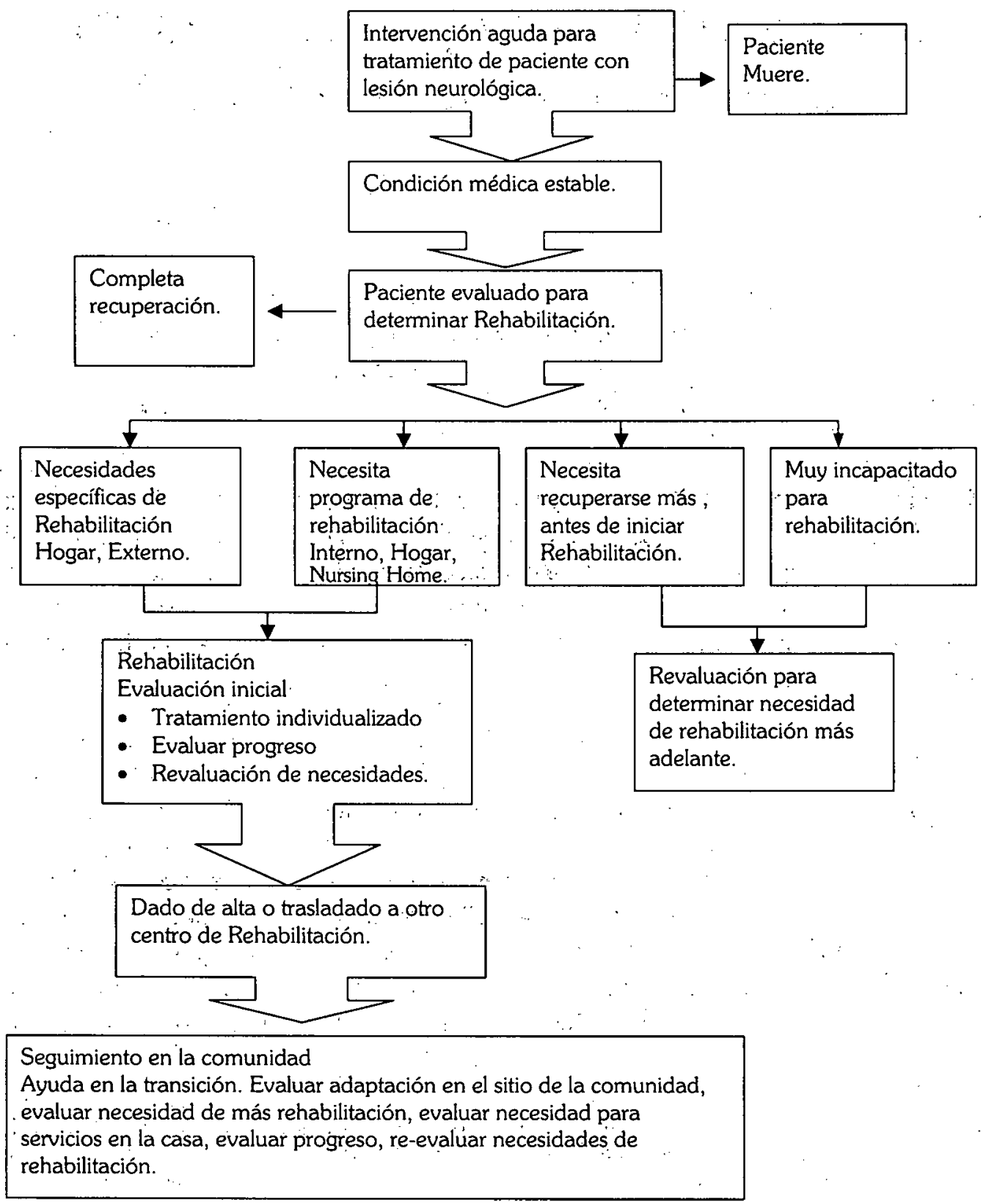


cual es determinada por una enfermera. Las visitas domiciliarias no son más de tres veces a la semana para fisioterapia, dos veces por semana para terapia ocupacional y probablemente ninguna visita de terapia del lenguaje. Esto es lo que ofrecen los seguros médicos tanto privados como del gobierno.

Actualmente los recursos para el paciente joven con ECV o TCE son limitados. No hay programas diseñados para esta población cuando los problemas físicos son mínimos y los problemas comportamentales y cognoscitivos son mayores. En Nueva York existen algunos programas de consulta externa que se utilizan para manejar estos pacientes. Sin embargo el gobierno es consciente que esta población no es atendida adecuadamente y esta haciendo investigaciones para tratar de desarrollar programas acordes a las necesidades de esta población.

En resumen podemos decir que la rehabilitación depende grandemente del aspecto económico y esto esta ligado a la capacidad o incapacidad que tiene la industria de rehabilitación, para demostrar los beneficios que tiene nuestra intervención.

Con esta idea en mente el gobierno americano y una universidad en Búfalo, Nueva York desarrollaron un proyecto con un médico rehabilitador, el cual tenía por objeto el desarrollo de una evaluación...

Una de las evaluaciones más utilizadas en el sistema Americano para determinar la intervención de rehabilitación, es una escala denominada FIM (Functional Independent Measure). Esta escala tiene como objetivo evaluar a los individuos a través de 18 ítems utilizando valores del 1 al 7. Estos ítems están divididos de acuerdo a las siguientes áreas:

1. Comer.

2. Aseo personal: lavarse dientes, peinarse o cepillarse el cabello, lavarse manos y cara, maquillarse o afeitarse.

3. Bañarse:lavarse, enjuagarse y cercarse el cuerpo del cuello para abajo excluyendo la espalda (puede ser en la tina o en la ducha).

4. Vestirse de la cintura para arriba.

5. Vestirse de la cintura para abajo.

6. Higiene en el baño: incluye el aseo del área perineal, ajustarse la ropa antes y después de utilizar el inodoro o el pato. 
7. Control de la vejiga.

8. Control de esfínter anal.

9. Traslados: a la cama, silla y/o silla de ruedas, o ponerse de pie para iniciar marcha como típico modo de marcha.

10. Traslados al baño.

11. Traslados a la tina o ducha.

12. Marcha y/o movilidad en silla de ruedas.

13. Escaleras.

14. Interacción Social.

15. Resolución de problemas.

15. Expresión.

16. Compresión.

17. Resolución de problemas.

18. Memoria.

Valores de la escala

Independiente

$7=$ Total independencia

$6=$ Independencia modificada

Dependencia

Dependencia modificada

$5=$ Supervisión

$4=$ Mínima ayuda $75 \%$ o más del esfuerzo

$3=$ Moderada ayuda 50-75\% del esfuerzo

Dependencia completa

2 = Máxima ayuda 25-50 del esfuerzo

1 = Tatal dependencia $0-25 \%$ del esfuerzo

Esta escala puede ser utilizada por cualquier profesional entrenado y requiere de la observación del paciente ejecutando estas actividades. El objetivo de la evaluación es determinar la cantidad de ayuda que otra persona debe proveer para que el paciente pueda realizar las actividades básicas cotidianas. Esto es importante tenerlo en cuenta porque esta evaluación no esta diseñada para evaluar cambios por ejemplo en cuanto a calidad de ejecución. Otra de las grandes limitaciones que tiene esta evaluación es que tiene efectos de techo. Esto significa que llega un punto 
en que la evaluación no muestra cambios a pesar que el paciente este demostrando que esta mejorando. Esto es evidente en el área cognoscitiva principalmente.

El beneficio que ha traído esta evaluación es que existe un banco de datos a nivel nacional en USA que ha permitido investigaciones. A pesar de las limitaciones conocidas, esta evaluación es utilizada en los Estados Unidos para investigar los resultados obtenidos en tratamientos en los diferentes hospitales. Utilizan los resultados en forma comparativa para determinar el costo beneficio de la intervención. Esto representa grandes problemas para los pacientes pues de esta evaluación depende la cantidad de ayuda que se le de al paciente en la casa o el tipo de tratamiento que se recomiende. Esto cuando el paciente a nivel cognoscitivo puede devolverse a vivir en su hogar, cuando no es este el caso, uno de los problemas que tiene esta evaluación es que no muestra la complejidad de las secuelas cognoscitivas, visuoperceptuales y emocionales de una lesión neurológica. Es aquí donde una muy buena documentación es la clave de un buen resultado para poder justificar la intervención de los profesionales en rehabilitación.

\section{Reintegro a la comunidad}

Vivir con las secuelas de una lesión neurológica es un reto para el resto de la vida. Un reto que implica la búsqueda constante de formas, para compensar o para adaptarse a los déficits que persisten. Para muchos de los sobrevivientes con lesiones neurológicas y sus familias el trabajo verdadero comienza después que la rehabilitación formal ha terminado. Uno de los trabajos más importantes que tenemos los que nos dedicamos a esta área ,es ayudar a preparar al cliente y su familia para este período de recuperación.

Muchos personas después de una lesión neurológica pueden vivir solos o con su familia. La mayoría requieren de una gran variedad de soportes. El impacto de cada lesión neurológica es individual, y cada persona y la familia tienen que desarrollar su camino único hacia la recuperación. Esta conferencia esta enfocada a los individuos que tienen personas que viven a su lado y pueden facilitar el proceso de reintegro a la comunidad.

\section{La transición}

Las primeras semanas después de dado de alta el paciente del programa de rehabilitación, son generalmente difíciles, pues el paciente intenta utilizar las recientemen- 
te aprendidas habilidades, pero sin el soporte del medio controlado que es un centro de rehabilitación. En la medida que pasan los días y la persona trata de reasumir sus roles, los déficits en las diferentes áreas de comunicación, motoras, cognoscitivas y emocionales se hacen más evidentes.

El poder manejar esta transición requiere de dos cosas: el estar preparado y de flexibilidad para realizar cambios y por consiguiente adaptarse. La familia como el paciente deben estar preparados afondo acerca de las diferentes situaciones a las cuales se pueden ver confrontados una vez regresen al hogar. De ser posible más de una visita domiciliaria debe ser ofrecida para trabajar el plan de reintegro al hogar y la comunidad. Actividades específicas deben ser discutidas para determinar como el paciente y la familia pueden realizarlas, pero simultáneamente debe trabajarse sobre lo inesperado, desarrollando en la familia y el paciente habilidades para resolver problemas y buscar ayuda cuando sea necesario para enfrentar las nuevas situaciones.

Es aquí donde un terapeuta ocupacional que trabaje en la casa con el paciente y la familia es indispensable.

\section{Factores para tener en cuenta}

\section{La salud de la persona encargada de cuidar al paciente. La forma en que el funcionamiento familiar puede verse afectado.}

Hay dos tipos de soporte; el proporcionado por la familia y el de la persona directamente encargada de cuidar al paciente. La presencia de estos dos tipos de soporte, facilita el alcanzar los mejores resultados de la rehabilitación a largo plazo.

Cuidar a una persona con secuelas de una enfermedad cerebro vascular o un trauma craneoencefálico puede ser una tarea de gran desgaste ya sea físico y/o emocional. En general investigaciones han demostrado que es mas fácil lidiar con limitaciones físicas que con limitaciones cognoscitivas o emocionales (Evans, 1986). Sin embargo existe un alto riesgo de fatiga y desgaste cuando el cuidado se tiene que proveer 24 horas al día 7 días de la semana. Es importante buscar medios para que el familiar encargado del paciente pueda tener tiempo de descanso. 
Las investigaciones también han demostrado efectos negativos en las personas dedicadas al cuidado de sobrevivientes de ECV ,tales como depresión, abuso de medicaciones para el paciente, frecuentes visitas al médico. (Blazer, Hughres and George, 1987; Kramer, German, Anthony, et al., 1985; Lichtenberg \& Barth, 1990; Schulttz, Uisintainer \& Williason, 1990).

Los aspectos culturales pueden tener un efecto importante, en relación a la actitud que se tenga acerca de lo que es incapacidad, minusvalía, el papel que debe desempeñar la familia en el proceso de transición y la habilidad que tenga la misma para lidiar con la complejidad de lo que es el sistema de salud.,

\section{Salud y promoción de salud durante el proceso de transición}

Disminuir el riesgo de un nuevo ECV a través de educación y promover un comportamiento saludable en cuanto a dieta, ejercicio, no fumar, etc.

\section{Prevención de caídas a través de identificar los factores de riesgo y los pasos necesarios para reducir los mismos}

Los esfuerzos del paciente por incrementar la movilidad, hacen que el peligro de caídas sea inevitable. El objetivo es minimizar el riesgo, mientras se promueven los esfuerzos del paciente por independizarse.

Riesgos remediables:

- Debilidad muscular.

- Equilibrio.

- Problemas con la visión y la percepción.

- Hipotensión ortostática.

- Uso de sedantes en exceso.

- Efectos secundarios de medicamentos.

- Equipo de ayuda mal prescrito o inadecuado, entrenamiento en el uso del mismo.

- Barreras medioambientales (tapetes, sillas, iluminación).

- Barreras arquitectónicas.

- Reasumir actividades recreacionales y valiosas para el paciente.

- Evaluar intereses y actividades que el individuo desarrollaba antes de la enfermedad. 
- Acordar con el paciente metas a corto y largo plazo.

- Revisar el valor que tienen las actividades y establecer la factibilidad de las mismas en relación a las capacidades actuales.

- Evaluación de equipo especial.

- Desarrollar estrategias para sobreponerse a las barreras que se encuentren en el hogar $y / o$ la comunidad.

- Encontrar nuevas actividades recreacionales que concuerden con los intereses del paciente y sus capacidades físicas.

- Educar al paciente y la familia en relación a los recursos de la comunidad.

- Cuando sea necesario, promover la creación de programas en la comunidad.

\section{Manejar carro}

La evaluación del paciente debe basarse en el aspecto neurológico, observaciones comportamentales, tests neuropsicológicos y visuoperceptuales.

\section{Volver al trabajo}

Los pacientes que se encontraban trabajando antes de tener el ECV, deben ser en lo posible evaluados nuevamente para reconsiderar su reintegro al trabajo. La orientación vocacional se debe recomendar.

Las barreras para un reintegro laborar pueden resumirse así:

- Falta de conocimiento de lo que es un ECV o un TCE y sus consecuencias.

- Barreras arquitectónicas en el puesto de trabajo.

- Acceso limitado a rehabilitación vocacional.

- Limitado conocimiento de los profesionales de la salud, acerca de los programas de rehabilitación vocacional.

- Actitudes negativas de algunos profesionales de la salud, en cuanto al reintegro laboral de los pacientes con ECV (por la edad).

- Actitudes negativas de los sobrevivientes, en cuanto a los servicios de rehabilitación vocacional, debido a la falta de conocimiento e información de los servicios existentes.

- Depresión y otros problemas emocionales que afectan la motivación y las habilidades del individuo para buscar trabajo. 
- Falta de incentivos económicos por parte de la cobertura de seguros médicos, pago de incapacidad, y costos de adaptaciones medioambientales y de transporte.

- Falta de interés o ineficiencia de los programas de rehabilitación vocaciónal para trabajar con estos pacientes.

Una vez hechos los delineamientos anteriores, cuyo objetivo es delienear aspectos importantes a tener en cuanta durante el proceso de reintegro a la comunidad del paciente con lesiones neurológicas (especialmente el paciente con ECV) se hace importante discutir que se sabe del tratamiento ofrecido a estos pacientes antes de ser dados de alta, y que se sabe su situación dentro de la comunidad.

A continuación discutiré brevemente algunos investigaciones que hacen referencia a este aspecto. En primer lugar, esta lo que algunos pacientes han comentado una vez salidos de la terapia, por ejemplo dicen haber participado en actividades por las cuales nunca habían demostrado interés o habían tenido que realizar antes (como cocinar), sin embargo el terapeuta ocupacional consideró que esta actividad era importante. Otros hicieron referencia a actividades básicas cotidianas como vestirse, la cual en ese particular momento de recuperación (período agudo) no consideraban importante, sin embargo fueron presionados para participar en la terapia.

En un estudio realizado por Brown y Bowen (1998) se evaluó en que medida el terapeuta ocupacional incluía al cliente y el ambiente dentro de su plan de tratamiento. Los investigadores encontraron que muchos de los objetivos a corto plazo no estaban relacionados con el objetivo descrito por el cliente inicialmente. También encontraron que en la mayoría de los caso se prefería la utilización de actividades simulados versus las reales. Ellos concluyen que este tipo de práctica se aleja de los modelos de intervención centrados en el cliente.

En el estudio realizado por Sabari, Meisler y Silver (2000), se recogió información con individuos que sufrieron un ECV pero que estaban radicados en la comunidad y participaban de un club para individuos con ECV. En este estudio etnográfico se encontraron dos temas importantes: preocupaciones en relación a necesidades no atendidas durante el proceso de rehabilitación y que los servicios fueron insuficientes para facilitar la transición a la comunidad. En este estudio hay comentarios que 
describe el tratamiento impersonal por parte del terapeuta, falta de atención al aspecto económico cuando se hacían sugerencias para modificar algún aspecto de la casa, falta de iniciativa para identificar las necesidades del paciente como por ejemplo practicar traslados al carro. Los autores concluyen que la rehabilitación del paciente con ECV debe ser una relación permanente entre el sobreviviente del ECV, la familia y los profesionales de rehabilitación. Los dos primeros deben dar a conocer sus necesidades y los profesiones deben proveer oportunidades a estos para que influencien el proceso de rehabilitación. Finalmente, sugieren que los profesionales de rehabilitación debe buscar maneras creativas y factibles para dar solución a los problemas identificados por los clientes.

Nilsson, Aniansson y Grmby (2000) estudiaron las necesidades de individuos que se encontraban en la comunidad después de dos años de haber sufrido ECV. En este estudio participaron 68 pacientes que fueron evaluados para determinar las necesidades de rehabilitación, el nivel de discapacidad, y la satisfacción en relación a varias situaciones de la vida. A pesar que el estudio identifica necesidades de intervención en actividades básicas cotidianas (identificadas a través del FIM), los hallazgos más importantes son las dificultades que el pacientes tienen para desarrollar actividades instrumentales de la vida diaria, como ir de compras, arreglar la casa y otras. Los individuos también expresaron no estar satisfechos con su salud y más aquellos que vivían solos. Una de las áreas identificadas como relevantes para tener una mejor calidad de vida fueron las actividades sociales.

\section{CONCLUSIÓN}

Después de esta breve revisión de algunos artículos que investigan la situación de individuos que sobreviven a un ECV, se hace clara la importancia de ir más allá de la intervención en actividades básicas cotidianas y actividades de la vida diaria. El sistema de salud y el gobierno deben proveer para estos individuos diferentes oportunidades para lograr un real reintegro a la comunidad y a la sociedad. Estos clientes describen su deseo de reanudar sus roles y su vida como un ser con valores individuales que puede aportar dentro de una comunidad.

(Lecturas recomendadas Reflections on doing, being and becoming De Ann Allart Wilcock). 


\section{REFERENCIAS}

Blazer, D., Hughes, D. \& George, L. (1987). The epidemiology of depression in the elderly community population. Gerontologist, 27, 281-287.

Brown, C. \& Bowen, R. (1998). Including the consumer and the environment in occupational therapy treatment planning. The Occupational Therapy Journal of Research, $18,44-62$

Evans, R. L. (1986). Caregiver compliance and feelings of burden in poststroke homecare. Psychol Rep, 59, 1013-1014.

Evans, R. L., Bishop, D. S. \& Dusley, R. T. (1992). Providing care to persons with diasability: effect on family caregivers. American Journal of Physical Medicine and Rehabilitation, 72, 144-147.

Gillen, G. \& Burkhardt, A. (1998) Stroke Rehabilitation. A functional based approach. Mosby; St. Louis: MO.

Kramer, M., German, P., Anthony, J. et el. (1985). Patterns of mental disorders among elderly residents of Eastern Baltimore. Journal American Geriatrics Soc, 33, 236245.

Lichtenberg, P.A. \& Gibson, T.A. (1992). Geriatric rehabilitation and the older adult family caregiver. Neurorehabilitation, 3, 62-71. http://www.tbims.org/combi/FAM/famsyl.html

Sabari, J., Meisler, J. \& Silver, E. (2000). Refelctions upon rehabilitation by member of a community based stroke club. Disability and Rehabilitation, 22, 330-336.

Shultz, R., Uisintainer, P. \& Williamson, G. (1990). Psychiatric and physical morbidity effects of caregiving. Journal Gerontolog Psych Sci, 45,181-191.

U.S. Department of Health and Human services (1995), Post Stroke Rehabilitation. AHCPR Publication No. 95-06622.

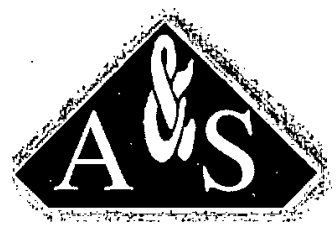

\section{Producciones Multimedia}

Diseño, Edición y desarrollo de proyectos multimedia para eventos empresariales universitarios escolares y familiares

Calle 69 No. 58 a 12 Tels.: 3143970 - 4829128 - 2508014 